\title{
Establish and Operate A District-Level Disease Surveillance Unit
}

\author{
Rajesh Kumar
}

\section{Introduction}

Integrated Disease Surveillance programme is a decentralized, state based surveillance programme in the country. It is intended to detect early warning signals of impending outbreaks and help initiate an effective response in a timely manner. It is also expected to provide essential data to monitor progress of on-going disease control Programme and help allocate health resources more efficiently. All outbreaks cannot be predicted or prevented. However, precautionary measures can be taken within the existing health infrastructure and service delivery to reduce risks of outbreaks and to minimize the scale of the outbreak if it occurs. The effectiveness with which national programmes are implemented and monitored, the alertness for identification of early warning signals and the capacity for initiating recommended interventions in a timely manner are important to achieve the above objectives.

The course of an epidemic is dependent on how early the outbreak is identified and how effectively specific control measures are applied. The epidemiological impact of the outbreak control measures can be expected to be significant only if these measures are applied in time. Scarce resources are often wasted in undertaking such measures after the outbreak has already peaked and the outcome of such measures in limiting the spread of the outbreak, and in reducing the number of cases and deaths, is negligible. When outbreaks occur or when the risk of such outbreaks is high, the co-operation of other government departments, non-governmental agencies and the community often becomes necessary. Such help will be more forthcoming if mechanisms for interactions have been developed before the onset of an outbreak.

The frequency of the occurrence of epidemics is an indication of the inadequacy of the surveillance system and preparedness to identify and control outbreaks in a timely manner.

\subsection{Public health surveillance?}

Surveillance is defined as the ongoing systematic collection, collation, analysis, and interpretation of data and dissemination of information to those who need to know in order that action is taken.

A more complete definition of surveillance is 'The ongoing systematic collection, analysis and interpretation of health data essential to planning, implementation, and evaluation of public health practice closely integrated with timely dissemination of these date to those who need to know.' The final link in the surveillance chain is the application of these data to prevention and control. A surveillance system includes a functional capacity for data collection, analysis and dissemination linked to public health programmes (CDC 1988).

Surveillance is the backbone of public health programme and provides information so that effective action can be taken in controlling and preventing diseases of public health importance. In some cases action must be immediate - within hours - in order to prevent large scale epidemics and deaths (Cholera, meningitis, food contamination).

In others, control and prevention activities are long term response to information about disease such as tuberculosis, HIV and Non Communicable Disease risk factors, for which action may be taken in weeks, months or even years.

\subsection{Key Elements of Surveillance System}

All surveillance systems involve six key elements:

- Detection and notification of health event

- Investigation and confirmation (Epidemiological, clinical, laboratory)

- Collection of data

- Analysis and interpretation of data

- Feedback and dissemination of results

- Response - a link to public health programme specially actions for prevention and control.

\subsection{Need of surveillance}

Uses of Surveillance:

- Recognize cases or cluster of cases to trigger interventions to prevent transmission or reduce morbidity and mortality 
- Assess the public health impact of health events or determine and measure trends

- Demonstrate the need for public health intervention programmes and resources and allocate resources during public health planning

- Monitor effectiveness of prevention and control measures

- Identify high-risk groups or geographical areas to target interventions and guide analytic studies

- Develop hypothesis that lead to analytic studies about risk factors for disease causation, propagation or progression.

\subsection{Integrated Disease Surveillance Programme (IDSP)}

The IDSP proposes a comprehensive strategy for improving disease surveillance and response through an integrated approach. This approach provides for a rational use of resources for disease control and prevention. In the integrated disease surveillance system:

_ the district level is the focus for integrating surveillance functions.

- all surveillance activities are coordinated and streamlined. Rather than using scarce resources to maintain vertical activities, resources are combined to collect Information from a single focal point at each level.

- Several activities are combined into one integral activity to take advantage of similar surveillance functions, skills, resources and target populations.

- The IDSP integrates both public and private sector by involving the private practitioners, private hospitals, private labs, NGOs, etc and also emphasis on community participation.

- The IDSP integrates communicable and non-communicable diseases. Common to both of them are their purpose in describing the health problem, monitoring trends, estimating the health burden and evaluating programmes for prevention and control.

- Integration of both rural and urban health systems as rapid urbanization has resulted in the health services not keeping pace with the growing needs of the urban populace. The gaps in receiving health information from the urban areas needs to be bridged urgently.

_ Integration with the medical colleges (both private and public) would also qualitatively improve the disease surveillance especially through better coverage.

\subsection{Objectives of the Integrated Disease Surveillance}

The overall general objective of the IDS is to provide a rational basis for decision-making and implementing public health interventions that are efficacious in responding to priority diseases. Keeping this in mind the main objectives of the IDS are:

- To establish a decentralized district-based system of surveillance for communicable and non-communicable diseases so that timely and effective public health actions can be initiated in response to health challenges in the urban and rural areas.

- To integrate existing surveillance activities (to the extent possible without having a negative impact on their activities) so as to avoid duplication and facilitate sharing of information across all disease control programmes and other stake holders, so that valid data are available for decision making at district, state and national levels.

To plan any disease control Programme and to identify and control outbreaks, it is important to know the following:

O Who gets the diseases?

O How many get them?

O Where do they get them?

$\mathrm{O}$ When do they get them?

O Why do they get them?

There are five steps in the surveillance procedure, which must be carried out at each level, starting from the Primary Health Centre (PHC). Each level must have the capacity for analyzing and using surveillance data for early detection, prevention and control of outbreaks. The five recommended steps are:

O Collection of data

O Compilation of data

$\mathrm{O}$ Analysis and interpretation

O Follow up action

O Feedback

\subsubsection{Pre-requisites for effective surveillance}

Prerequisite for effective surveillance are:

$O$ Use of standard case definitions.

O Ensure regularity of the reports. 
O Action on the reports For developing an effective disease surveillance system, the District Health Officer/ PHC Medical Officer must also be clear about:

O What information to gather?

O How often to compile and analyze the data?

O How often and to whom to report?

O What Performa or formats to use?

O What action to take?

The data collected should be uniform, regular and timely. Standard case definitions are important to ensure uniformity in reporting so that all reporting units use the same criteria for reporting cases. It is also important to have a list of all reporting units so that the regularity and timeliness of the reports is checked. If no cases are seen, a nil report should be submitted. All levels in the system must:

o Have the standard case definitions?

o Have a list of all reporting units?

o Monitor receipt of reports in time

o Monitor completeness of reports

The standard case definition of diseases is given in a separate manual. Depending on the level of expertise and specificity, disease surveillance in IDSP will be of following three categories:

\subsection{Methods of data collection}

Several methods can be used for collecting data. While routine reporting (passive surveillance) is universalized, other methods are need and area specific. These include:

I Sentinel surveillance

Ii Active surveillance (active search for cases)

Iii Vector surveillance

IV Laboratory surveillance

V Sample surveys

VI Outbreak investigations

Vii Special studies

The report development objective is to improve the information available to the government health services and private health care providers on a set of high-priority diseases and risk factors, with a view to improving the on-the-ground responses to such diseases and risk factors through Information \& Communication Technology in District Bhiwani. Specifically, the reports aim:

- To establish a decentralized District based computerized system of surveillance for communicable and noncommunicable diseases, so that timely and effective public health actions can be initiated in response to health challenges in the District.

- To improve the efficiency of the existing surveillance activities of disease control programs and facilitate sharing of relevant information with the health administration, community and other stakeholders so as to detect disease trends over time and evaluate control strategies.

The report will assist the health administration of District Bhiwani to:

- surveil a limited number of health conditions and risk factors;

- strengthen data quality, analysis and links to action;

- improve laboratory support;

- train stakeholders in disease surveillance and action;

- coordinate and decentralize surveillance activities;

- Integrate disease surveillance at the district level and Community Health Centre/Primary Health Centre/SubCentre levels, and involve communities and other stakeholders, particularly the private sector.

\section{Research Design}

A research design is the overall framework of the plan used for the collection and analysis of data collected. Research design includes problem definition, a plan for gathering data, analysis of data, time framework and budget estimate.

The research design presents a organized and systematic to the formulation and implementation of the research report. In fact a suitable research design guards against collection of the irrelevant data and therefore results are more economical. 


\section{Type of Research}

This research is descriptive and diagnostic type. Descriptive research study describes the characteristics of a particular individual or a group and diagnostic research studies determines the frequency with which something occurs or its association with something else.

\section{Sample Selection and Size}

\section{Research Methodology}

In the most research design it become almost impossible to examine the entire universe. So the only alternative is to resort to sampling. This is true for present study as well. A basic principle to be followed in sampling is that the sample chosen must be representative of the entire universe to be studied. In this research study the sample size of 100-125 respondents has been taken.

The sampling was done using convenience selection based on random sampling and the target respondent was any reporting person i.e. Health worker/LHV/ANM/MO/SMO/Private practitioner etc. Bhiwani city was chosen as the centre of the study, leading to an overall sample size of 100-125 respondents are generally chosen from the General Hospitals/CHC/PHC/SC/Private Hospitals.

\section{Sources of Data}

This phase is generally the most expensive and most liable to error. The task of data collection begins after a research problem has been defined and the research plans checked out. For this purpose the researcher has to keep in mind two types of data viz. primary and secondary.

\section{PRIMARY DATA:}

The data which are collected is fresh and for first time and thus happen to be original in character. There are several methods of collecting primary data. Important ones are: observation method, interview method, through questionnaires, through schedules etc.

\section{SECONDARY DATA:}

The data which has been collected by someone else and which have already been passed through the statistical process. Secondary data means data that are already available i.e. they refer to the data which have already been collected and analyzed by someone else. The sources of secondary data are: various publications, magazines, books, newspapers IEC material etc.

\section{Selection Of Appropriate Method For Data Collection:}

There are various methods of data collection. Such as: Nature, scope and object of enquiry, availability of funds, time factor, precision required etc.

So far as the present study is concerned primary data and secondary data both are used. Primary source of data are personal interview with the help of questionnaire at various hospitals. During survey, each respondent was given a set of questions and their behavior is checked out. The secondary source of data are the different manuals of health department, IEC material which are printed time to time by the Government, study material of health department and the most powerful source is Internet.

\section{DATA ANALYSIS}

The collected data was arranged in the form of tables so that meaningful inference could be drawn out of the data. Subsequently, the tabulated data was analyzed with the help of diagrams and tables and histograms and conclusions were drawn on the basis of the result so generated. 